\title{
Turbulent Rayleigh-Bénard convection under strong non-Oberbeck-Boussinesq conditions
}

\author{
Hiufai Yik $\odot,{ }^{1, *}$ Valentina Valori $\odot,{ }^{2,3,4}$ and Stephan Weiss $\oplus^{1,5, \dagger}$ \\ ${ }^{1}$ Max Planck Institute for Dynamics and Self-Organization, Am Fassberg 17, D-37077 Göttingen, Germany \\ ${ }^{2}$ Department of Radiation Science and Technology, Faculty of Applied Sciences, Delft University of \\ Technology, Mekelweg 15, 2629 JB Delft, The Netherlands \\ ${ }^{3}$ Process and Energy Department, Delft University of Technology, Mekelweg 2, \\ 2628 CD Delft, The Netherlands \\ ${ }^{4}$ Institut für Thermo-und Fluiddynamik, Technische Universität Ilmenau, \\ Postfach 100565, D-98684 Ilmenau, Germany \\ ${ }^{5}$ Max Planck - University of Twente Center for Complex Fluid Dynamics
}

(Received 29 May 2020; accepted 10 September 2020; published 20 October 2020)

\begin{abstract}
We report on Rayleigh-Bénard convection with strongly varying fluid properties experimentally and theoretically. Using pressurized sulfur-hexafluoride $\left(\mathrm{SF}_{6}\right)$ above its critical point, we are able to make measurements at mean temperatures $\left(T_{m}\right)$ and pressures $\left(P_{m}\right)$ along Prandtl-number isolines in the $(T, P)$ parameter space. This allows us to keep the mean Rayleigh- $\left(\mathrm{Ra}_{m}\right)$ and Prandtl number $\left(\operatorname{Pr}_{m}\right)$ constant while changing the temperature dependences of the fluid properties independently, e.g., probing the liquidlike or gaslike region that are left and right of the supercritical isochore. Hence, non-Oberbeck-Boussinesq (NOB) effects can be measured and analyzed cleanly. We measure the temperature at midheight $\left(T_{c}\right)$ as well as the global vertical heat flux. We observe a significant heat transport enhancement of up to $112 \%$ under strong NOB conditions. Furthermore, we develop a theoretical model for the global vertical heat flux based on ideas of Grossmann and Lohse (GL) in OB systems, adjusted for nonconstant fluid properties. In this model, the NOB effects influence the boundary layer and hence $T_{c}$, but the change of the heat flux is predominantly due to a change of the fluid properties in the bulk, in particular the heat capacity $c_{p}$ and density $\rho$. Predictions from our model are consistent with our experimental results as well as with previous measurements carried out in pressurized ethane and cryogenic helium.
\end{abstract}

DOI: 10.1103/PhysRevFluids.5.103502

\section{INTRODUCTION}

Rayleigh-Bénard convection (RBC), where a horizontal fluid layer of height $H$ is heated from below and cooled from above, is often studied under the Oberbeck-Boussinesq (OB) approximation, which assumes constant fluid properties except for the density, which changes linearly with

\footnotetext{
*hyik@ds.mpg.de

†stephan.weiss@ds.mpg.de
}

Published by the American Physical Society under the terms of the Creative Commons Attribution 4.0 International license. Further distribution of this work must maintain attribution to the author(s) and the published article's title, journal citation, and DOI. Open access publication funded by the Max Planck Society. 
temperature, constituting buoyant driving [1-3]. Under such simplification, the system is controlled by only two dimensionless parameters, namely the Rayleigh number, $\mathrm{Ra}=\alpha g \Delta H^{3} /(\kappa v)$, and the Prandtl number, $\operatorname{Pr}=v / \kappa$. Here, $\Delta=T_{b}-T_{t}$ is the temperature difference between bottom $\left(T_{b}\right)$ and top $\left(T_{t}\right), g$ is the gravitational acceleration, $\alpha$ is the thermal volume expansion, $v$ is the kinematic viscosity, and $\kappa$ is the thermal diffusivity. Numerous models and predictions on global scaling and turbulent characteristics were developed for turbulent RBC under OB conditions in recent years [4].

In many natural and industrial systems, however, large temperature and pressure differences occur, resulting in variations of fluid properties throughout the cell and a violation of the OB approximation. For example, the density of plasma inside the solar convection layer has a $10^{5}$ fold discrepancy, which hampers current Rayleigh-Bénard models being applied [5]. In industrial applications, supercritical fluids with their large heat capacity and small viscosity are of great interest for efficient heat transport, as, for instance, in coal-fueled or nuclear power plants of Generation IV [6-8]. Furthermore, using supercritical fluids in power plants permits to operate at larger temperatures, which helps to increase the thermal efficiency of the cooling circuit drastically compared to two phase flows.

However, the fluid properties of supercritical fluid are particularly sensitive to temperature and pressure variations, and thus no longer constant throughout the system. It is therefore important to understand how such nonconstant fluid properties effect the flow field and the thermal transport properties of these systems and how current models need to be adapted for the non-OB cases.

Non-OB (NOB) convection has been studied in the past, both experimentally and numerically. The two most important responses are (i) the horizontally averaged center temperature at midheight, $T_{c}=\langle T(x, y, z=0.5 H)\rangle_{x, y, t}$, and (ii) the global vertical heat flux. The former parameter measures the up-down asymmetry of the system, while the latter is a global easily measurable quantity that is particular important in industrial applications and natural systems.

While NOB-convection close to convection onset (i.e., for laminar flows) is somehow understood [9], for turbulent convection - despite some effort - the results are sparse and so far no simple conclusion can be drawn. This is to some extent due to the large amount of control parameters that become relevant in NOB-convection (see, e.g., [10]).

In general, the variations of fluid parameters between the warm bottom and the cold top destroy the up-down symmetry of the system, resulting in $T_{c} \neq T_{m}$, with $T_{m}$ being the average temperature $T_{m}=\left(T_{t}+T_{b}\right) / 2$. For liquids, $T_{c}>T_{m}$ was observed [11-17], while $T_{c}<T_{m}$ was found for gases as the working fluid [14,18-21]. Various models predicting $T_{c}$ as a function of $\Delta, T_{m}$, and fluid parameters have been proposed and predict $T_{c}$ more or less accurately. Most of the models consider the boundary layers at the top and bottom. For example, Wu and Libchaber [18] proposed that either the temperature scale, a velocity scale, or a corresponding boundary Rayleigh number evaluated at the bottom and top have to match, leading to three different models for $T_{c}$. Ahlers et al. [14,19] assumed laminar boundary layers at the bottom and top and solved numerically the momentum and heat equation assuming temperature-dependent fluid properties, while neglecting buoyancy. There is also the virtual cell model [20] that predicts $T_{c}$ by assuming that the top part and the bottom part of the convection system can be described as two separate OB systems that both follow the same $\mathrm{Nu}(\mathrm{Ra})$ relation.

While different observations on the influence of NOB effects on $T_{c}$ are in good agreement, results for the heat transport in NOB convection are not conclusive and at first seem contradicting. Measurements in pressurized gases usually show an increase in the vertical heat flux under NOB conditions, as shown in [14,19,22,23]. Investigations using water resulted in a heat transport reduction of just a few percent compared to the OB case [11,15], whereas investigations using glycerol [13,16,17] and air at atmospheric pressure [21] do not show any clear trend, despite a significant change in $T_{c}$.

While there are a handful of models developed to predict $T_{c}$ in NOB fluids [11,12,18-20], there is a wide research gap in modeling the global heat flux theoretically. For example, the extended mixing zone model by $\mathrm{Wu}$ and Libchaber [18] is based on the same assumption that led to a $\mathrm{Nu} \propto \mathrm{Ra}^{2 / 7}$ scaling in the OB case, which is experimentally observed only in a rather narrow range of Ra and 
Pr. Hence, the same model is not expected to hold in general for NOB systems. The model proposed by Ahlers et al. [11] based on the temperature gradient at the sidewalls of the cell assumes that the viscous boundary layer thickness is unchanged, which is not expected to hold in strong NOB systems. And the virtual cell model [20] only predicts $T_{c}$ but not the heat flux. So far, none of these models is available to explain the strong global heat flux enhancement mentioned above.

In the next section, we introduce the governing equations and the most important dimensionless parameters. In Sec. III and IV, we describe our measurement scheme and the experimental setup. In Sec. V, we present our theoretical model for the vertical heat flux for NOB fluids, and we compare it with our measurements and the results of others. The paper ends with a conclusion in Sec. VI.

\section{DIMENSIONLESS PARAMETERS}

In the NOB system, next to $\mathrm{Ra}$ and $\mathrm{Pr}$, also the temperature $(T)$ and pressure $(P)$-dependent fluid properties determine the state of the system. Here, we only consider $T$-dependent fluid properties and neglect spatial variations of $P$. Thus, we write the fluid properties as $\mathbf{X}\left(T, P_{m}\right) \equiv$ $\mu\left(T, P_{m}\right), \Lambda\left(T, P_{m}\right), \alpha\left(T, P_{m}\right), \rho\left(T, P_{m}\right), c_{p}\left(T, P_{m}\right)$, where $\mu$ is the dynamic viscosity, $\Lambda$ is the thermal conductivity, $\alpha$ is the isobaric expansion coefficient, $\rho$ is the density, and $c_{p}$ is the isobaric heat capacity, evaluated at a given pressure $P_{m}$. Usually a violation of the OB approximation results in changes of $T_{c}$ and the vertical heat flux $q$.

Equations for momentum, temperature, and mass can be written as

$$
\begin{gathered}
\rho \frac{\partial}{\partial t} u_{i}+\rho u_{j} \frac{\partial}{\partial x_{j}} u_{i}=-\frac{\partial}{\partial x_{i}} p+\frac{\partial}{\partial x_{j}}\left(\mu \frac{\partial}{\partial x_{j}} u_{i}\right)-g\left(\rho-\langle\rho\rangle_{V}\right) \delta_{i z} \\
c_{p} \rho \frac{\partial}{\partial t} T+c_{p} \rho u_{i} \frac{\partial}{\partial x_{i}} T=\frac{\partial}{\partial x_{j}}\left(\Lambda \frac{\partial}{\partial x_{j}} T\right) \\
\frac{\partial}{\partial t} \rho+\frac{\partial}{\partial x_{j}}\left(\rho u_{j}\right)=0
\end{gathered}
$$

where the indices $i, j$ denote the horizontal $(x, y)$ and vertical $(z)$ coordinates, $\langle\cdots\rangle_{V}$ is the volume average, and $p=P+\int_{z_{0}}^{z} \rho\left(z^{\prime}\right) g d z^{\prime}$. One can show [10] that by introducing proper length, time, and temperature scales, $\tilde{x}_{i} \equiv x_{i} / H, \tilde{t} \equiv t /\left(H / \sqrt{\alpha_{m} g \Delta H}\right), \tilde{u}_{i} \equiv u_{i} / \sqrt{\alpha_{m} g \Delta H}, \theta \equiv\left(T-T_{\text {top }}\right) / \Delta, \tilde{\mathbf{X}} \equiv$ $\mathbf{X} / \mathbf{X}_{m}$, where $\mathbf{X}_{m} \equiv \mathbf{X}\left(T=T_{m}\right)$, the system is determined by the OB control parameters evaluated at $\left(T_{m}, P_{m}\right)$, i.e., $\operatorname{Ra}_{m} \equiv \alpha_{m} g \Delta H^{3} / v_{m} \kappa_{m}, \operatorname{Pr}_{m} \equiv v_{m} / \kappa_{m}$, and by the equation of states for the fluid properties, $\tilde{\mathbf{X}}\left(\theta, T_{m}, P_{m}\right)$.

As mentioned already, next to the averaged temperature at midheight $\left(T_{c}\right.$ or $\left.\theta_{c}\right)$, the global vertical heat flux $q$ is the most important response parameter. It is usually expressed dimensionless as the Nusselt number $\mathrm{Nu}=q / q_{\text {cond }}$, with $q_{\text {cond }}$ being the heat transport that would occur without convection. While for the OB case the heat conductivity $\Lambda$ is constant and hence it is simply $q_{\text {cond }}=q_{\mathrm{OB}}=\Lambda_{m} \Delta / H$, for the NOB case, i.e., when $\Lambda$ depends on $T$, one needs to integrate over the entire conductivity profile as shown in [24], $q_{\text {cond }}=H^{-1} \int_{T_{t}}^{T_{b}} \Lambda(T) d T$.

For the analysis presented in Sec. V, we define a mean Nusselt number as $\mathrm{Nu}_{m}=q / q_{\mathrm{OB}}$, which compares the heat flux with the conductive heat flux in the OB case. This is only for simplicity later on, and since $\mathrm{Nu}=\mathrm{Nu}_{m} q / q_{\text {cond }}$ both Nusselt numbers can easily be converted into each other.

In a very similar way, we will also define a mean Reynolds number as $\operatorname{Re}_{m} \equiv U H / v_{m}$. Here, $U$ is the characteristic velocity scale in the turbulent bulk, i.e., the velocity of typical eddies of the size of the energy injection scale. While we cannot measure velocities in our setup, $\operatorname{Re}_{m}$ is an important global response parameter in turbulent $\mathrm{RBC}$, and as such it will play a role later on in Sec. V.

The definitions for $\mathrm{Nu}_{m}$ and $\mathrm{Re}_{m}$ are chosen to be consistent with our normalized fluid properties, i.e., $\tilde{\mathbf{X}}=\mathbf{X} / \mathbf{X}_{m}$. 
(a)

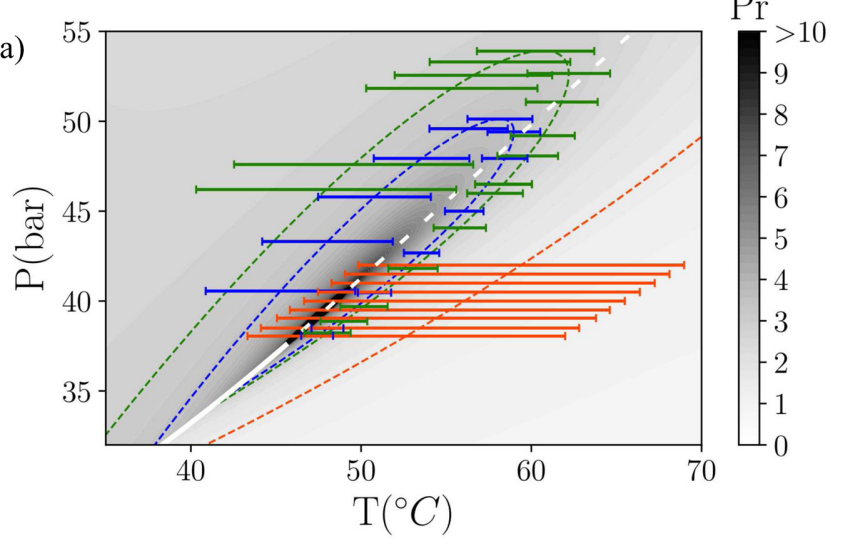

(b)

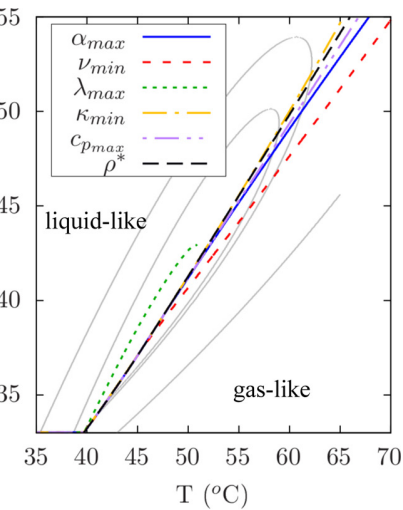

FIG. 1. (a) Pressure-temperature phase space for $\mathrm{SF}_{6}$ with the vapor pressure (solid white line), the supercritical isochore (white dashed line), and different isocontour lines of $\operatorname{Pr}$ (dashed line). Their color represents $\operatorname{Pr}_{m}=3.5$ (blue), 3.0 (green), and 1.5 (orange). Horizontal lines indicate the temperature range $\left\{T_{t}, T_{b}\right\}$ at which experiments were conducted. For all measurements it was $\mathrm{Ra}_{m}=2.8 \times 10^{11}$. (b) $P$ - $T$ phase space with the lines of extreme values, i.e., where $\partial \alpha / \partial T=0, \partial \nu / \partial T=0, \partial \lambda / \partial T=0, \partial \kappa / \partial T=0, \partial c_{p} / \partial T=0$, as well as the supercritical isochore $\rho=\rho^{*}$. These lines separate the liquidlike from the gaslike region. We also show the isolines of $\operatorname{Pr}=1.5,3.0$, and 3.5 as gray lines for comparison.

\section{EXPERIMENTAL PROGRAM AND THE DESCRIPTION OF THE SETUP}

For quantitatively comparing $\mathrm{Nu}_{m}$ in NOB and $\mathrm{OB}$ convection, the fluid properties $\tilde{\mathbf{X}}$ should be varied while keeping $\operatorname{Ra}_{m}$ and $\operatorname{Pr}_{m}$ fixed. This is nontrivial and has so far not been done experimentally because $\operatorname{Pr}_{m}$ and $\tilde{\mathbf{X}}$ are both intrinsic properties of a fluid. Previous experiments and simulations have been conducted at fixed $T_{m}$ with varying $\tilde{\mathbf{X}}$ by changing $\Delta$ and $P_{m}$, thus causing also changes in $\operatorname{Ra}_{m}$ and $\operatorname{Pr}_{m}$ [11,14,17,19,25,26]. Manipulating the degree of the NOB effect in such a way always results also in a change of $\mathrm{Ra}_{m}$, which also changes the heat flux. Thus, any observation of heat flux changes is difficult to interpret because the non-uniform fluid properties, i.e. $\tilde{\mathbf{X}}(\theta)$ is not the only independent variable. Furthermore, changing $\Delta$ at a specific $T_{m}$ and $P_{m}$ also limits the shape of $\tilde{\mathbf{X}}(\theta)$ to be explored as part of the profile is always being retained in the same set of experiments.

Here, we use the fact that one can draw contour lines of constant $\operatorname{Pr}_{m}$ in the $T$ - $P$ - phase diagram near the critical point $\left(T^{*}, P^{*}\right)$ of a fluid as shown in Fig. 1. We perform experiments along these contour lines using sulfur hexafluoride $\left(\mathrm{SF}_{6}\right)$ as the working fluid by varying $T_{m}$ and $P_{m}$ and adjusting $\Delta$ such that both $\operatorname{Pr}_{m}$ and $\mathrm{Ra}_{m}$ are kept constant for a set of experiments (horizontal lines in Fig. 1). In this way, one can not only change the strength of the NOB effects, i.e., the difference between the fluid properties at the warm bottom and the cold top, but one can even change the monotonic behavior of $\mathbf{X}(T)$. For example, at small $P$ and large $T$, many fluid properties behave similarly to gases $[\alpha$ decreases while $v$ and $\kappa$ increase with increasing $T$; see also Fig. 1(b) and Fig. 2(a)]. For large $P$ and small $T$, on the other hand, the monotonic behavior of the fluid properties is similar to that of liquids [ $\alpha$ increases while $v$ and $\kappa$ decrease with increasing $T$; see also Fig. 1(b) and Fig. 2(b)].

We note that the gaslike and liquidlike areas in the supercritical phase space are not separated by a sharp line, but rather by a wedgelike transition area [shown in Fig. 1(b)]. This is because lines of extreme values are different for different fluid properties. In this region, for example, the kinematic viscosity $v$ might decrease with $T$ as for liquids, whereas the expansion coefficient $\alpha$ decreases as well with $T$, as is the case for gases. We make measurements in the liquidlike, the gaslike, as well as the transition region while keeping $\operatorname{Pr}_{m}$ and $\mathrm{Ra}_{m}$ constant. 
(a)
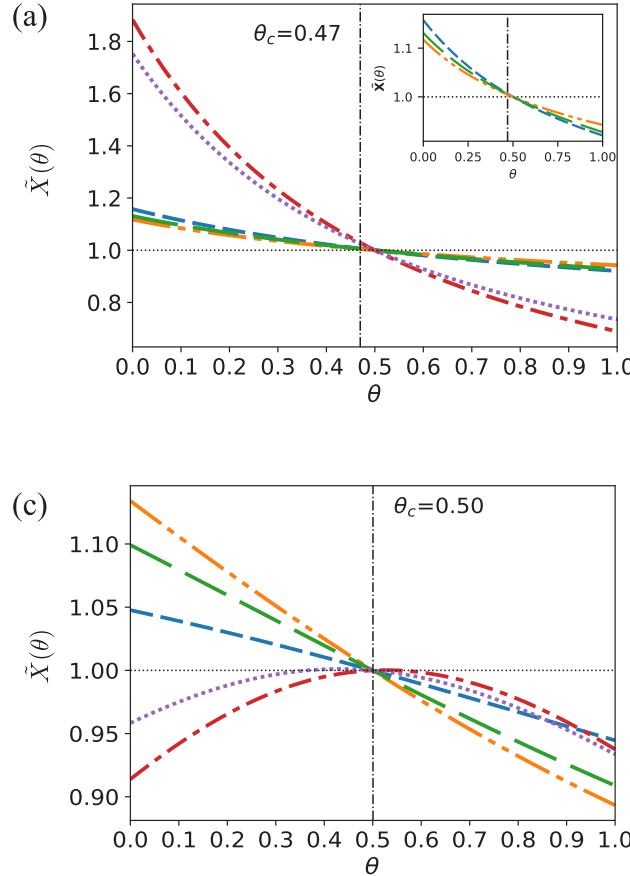

(b)

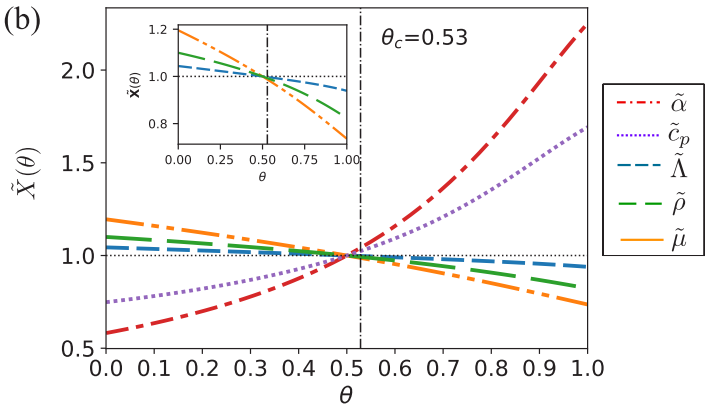

(d)

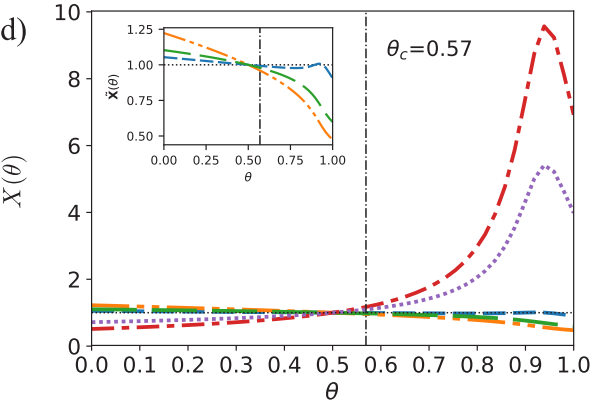

FIG. 2. Normalized fluid properties $\tilde{\mathbf{X}}$ (see legend) as a function of the normalized temperature $\theta$ for four representative experimental runs (i.e., $T_{m}, P_{m}$ ) and $\operatorname{Pr}_{m}=3.5$. Vertical dash-dotted line: experimentally measured $\theta_{c}$. Horizontal dotted line: $\tilde{X}(\theta)=1$, i.e., the OB case. Inset: zoomed-in plot showing $\tilde{\rho}, \tilde{\Lambda}, \tilde{\mu}$. (a) Gaslike fluid at $T_{m}=50.79^{\circ} \mathrm{C}$ and $P_{m}=40.47$ bar. (b) Liquidlike fluid at $T_{m}=50.789^{\circ} \mathrm{C}, P_{m}=45.79$ bar. (c) Fluid with the most OB-like properties, i.e., slightly above the critical isochore at $T_{m}=58.98{ }^{\circ} \mathrm{C}$ and $P_{m}=49.41$ bar. (d) Mostly liquidlike fluid at $T_{m}=45.26^{\circ} \mathrm{C}$ and $P_{m}=40.56$ bar.

For a better illustration, we show in Fig. 2 the profiles $\tilde{\mathbf{X}}(\theta)$ for five important fluid properties and at four typical experimental conditions. We see that some parameters, like $\tilde{\rho}, \tilde{\Lambda}$, and $\tilde{\mu}$, show a monotonic decrease for all conditions. However, for ratios such as $\kappa=\Lambda /\left(c_{p} \rho\right)$ or $v=\mu / \rho$ (best seen in the insets) or derivates like $\tilde{\alpha}$ and $\tilde{c}_{p}$, we observe opposite monotonic trends in the gaslike [Fig. 2(a)] and liquidlike regions [Fig. 2(b)]. Figure 2(c) shows fluid profiles at a point farthest away from the critical point, where fluid properties are nearly symmetric and the system is closest to an OB system. Very often in the experiment, we have a situation as in Fig. 2(d). The fluid is mostly liquidlike, but the temperature at the warm bottom plate crosses slightly the lines of maximal $\tilde{\alpha}$ and $\tilde{c}_{p}$. Both values, however, are still larger at the bottom plate than at the top, which will result in an increase of $\theta_{c}$ similar to liquids.

For the study presented here, we have carried out experiments at fixed $\mathrm{Ra}_{m}=2.8 \times 10^{11}$ and $\operatorname{Pr}_{m}=1.5,3.0,3.5$. In each set of experiments for a given $\operatorname{Pr}_{m}, \tilde{\mathbf{X}}(\theta)$ is changed by going along the isocontour line of $\operatorname{Pr}_{m}$ from the gaslike (small $P_{m}$ and $\rho_{m}$ ) to the liquidlike side (large $P_{m}$ and $\rho_{m}$ ).

The experimental setup is similar to the one used in $[14,19]$. The cylindrical convection cell of diameter $D=10.16 \mathrm{~cm}$ and height $H=5.08 \mathrm{~cm}(\Gamma=D / H=2)$ has stainless steel sidewalls and copper top and bottom plates. The cell is placed in a dry container filled with thermal insulating foam surrounded by a temperature-regulated circulating water bath that cools the top plate to its desired temperature. The cell is connected to a temperature-regulated gas bottle through a capillary tube for pressure regulation. Two thermistors were placed in each of the top and bottom plates roughly a millimeter away from the fluid and roughly $2 \mathrm{~cm}$ away from the cylinder axis. In addition, four 
thermistors were placed outside of the sidewall at midheight for measurements of $T_{c}$. Each measurement lasted $24 \mathrm{~h}$ with the top and bottom plate temperature being kept constant at their desired values within $0.05 \mathrm{~K}$ and pressure within 0.02 bar. The heat transport was measured via the Ohmic heat dissipation into the bottom plate heater $Q_{\text {heater }}$. However, not all of the heat was transported from the bottom to the top by the turbulent flow, but there was a significant heat flux through the insulation material into the surrounding water bath $\left(Q_{\text {loss }}\right)$. We determined $Q_{\text {loss }}$ as a function of $\Delta$ by conducting measurements in an evacuated cell for different $\Delta$ and fitting a quadratic function to the measured sampling points. To obtain the correct heat flux between the bottom and top plate $Q$, we corrected the measured Ohmic heat input accordingly, $Q=Q_{\text {heater }}-Q_{\text {loss }}$. In our experiment, $Q_{\text {loss }}$ can account for up to $20 \%$ of $Q_{\text {heater }}$.

We note that this correction also accounts for the heat transport inside the stainless steel sidewall. This contribution, however, is significantly smaller than the heat flux through the insulation material, and it accounts for not more than a few percent of the total $Q_{\text {loss }}$. We also note that there are additional, higher-order contributions for the heat transport through the sidewall (see [27,28]) for which we do not account. These contributions result from different thermal gradients in the fluid and in the sidewall due to its high thermal conductivity. As a consequence, the sidewall damps temporal and spatial fluctuations of the fluid close to it and acts as a bypass for the heat flux close to the top and bottom plate. Correcting these higher-order contributions is a nontrivial task that requires numerical simulations. We refrain from doing so because such corrections potentially introduce other uncertainties and also because we always compare our results with the OB case and hence we expect the influence of the sidewall on this deviation to be negligible, and deviations of $\mathrm{Nu}_{m}$ and $T_{c}$ due to NOB effects can be captured reliably.

As pointed out before, we use $\mathrm{SF}_{6}$ as the working fluid. $\mathrm{SF}_{6}$ has an experimentally convenient critical point at $T^{*}=45.6^{\circ} \mathrm{C}$ and $P^{*}=37.6$ bar, it has a high density (hence a small $v$ and $\kappa$ ), and subsequently it enables experimentation at larger Ra. However, most importantly, its fluid properties close to the critical point have been studied extensively and are well known. We use the fluid properties reported by NIST (REFPROP database [29]) in our analysis.

\section{EXPERIMENTAL RESULTS}

Figure 3 shows measurements of $\theta_{c}$ and $\mathrm{Nu}_{m}$ as a function of the normalized density $\rho_{m} / \rho^{*}$ for different $\operatorname{Pr}_{m}$. The vertical dashed lines denote $\rho_{m} / \rho^{*}$ for which $\theta_{c}=0.5$, i.e., where the fluid is expected to be most OB. The relative density $\rho_{m} / \rho^{*}$ is chosen as the control parameter as it is best suited to represent the monotonic change of the NOB effects. At small $\rho_{m} / \rho^{*}$, the system is close to its critical point (i.e., large NOB effects) but the fluid properties have a gaslike temperature dependency. With increasing $\rho_{m} / \rho^{*}$, the system moves further away from the critical point, and thus closer to the OB system. There, the fluid properties depend only weakly on $T$ and are nearly symmetric between bottom and top. With increasing $\rho_{m} / \rho^{*}$, the fluid properties behave more liquidlike and the NOB effects become stronger as the system gets closer to its critical point.

The first column in Figs. 3(a), 3(c) and 3(e) shows measurements of $\theta_{c}$ as a function of $\rho_{m} / \rho^{*}$ (black crosses). We see that $\theta_{c}$ increases monotonically with increasing $\rho_{m} / \rho^{*}$ and that in general it is $\theta_{c}<0.5$ in gaslike $\left(\rho_{m} / \rho^{*}<1\right)$ and $\theta_{c}>0.5$ in liquidlike $\left(\rho_{m} / \rho^{*}>1\right)$ fluids. This observation is consistent with previous measurements in liquids (e.g., [11]) and gases (e.g., [14,19]). Note that the point with $\rho_{m} / \rho^{*} \approx 1$ is not the point where the system is closest to an OB system, as can clearly be seen in Fig. 1 by comparing the points where the Pr-isolines have the strongest curvature and the critical isochore of the system (white dashed line). As a result, $\theta_{c}=0.5$ is observed for $\rho_{m} / \rho^{*}$ slightly larger than 1 .

We compare our results with different models for $\theta_{c}$, and we find that the third model by Wu and Libchaber (mixing zone model) [18] and the virtual-cell model [20] hold well in a weakly NOB system, but they fail at strongly NOB systems [30]. This is because both models assume characteristic temperatures for the top and bottom boundary layers, which is not enough to describe a rather complex function $\tilde{\mathbf{X}}\left(\theta, T_{m}\right)$ inside the BL. The extended-boundary-layer theory $[14,19]$, 

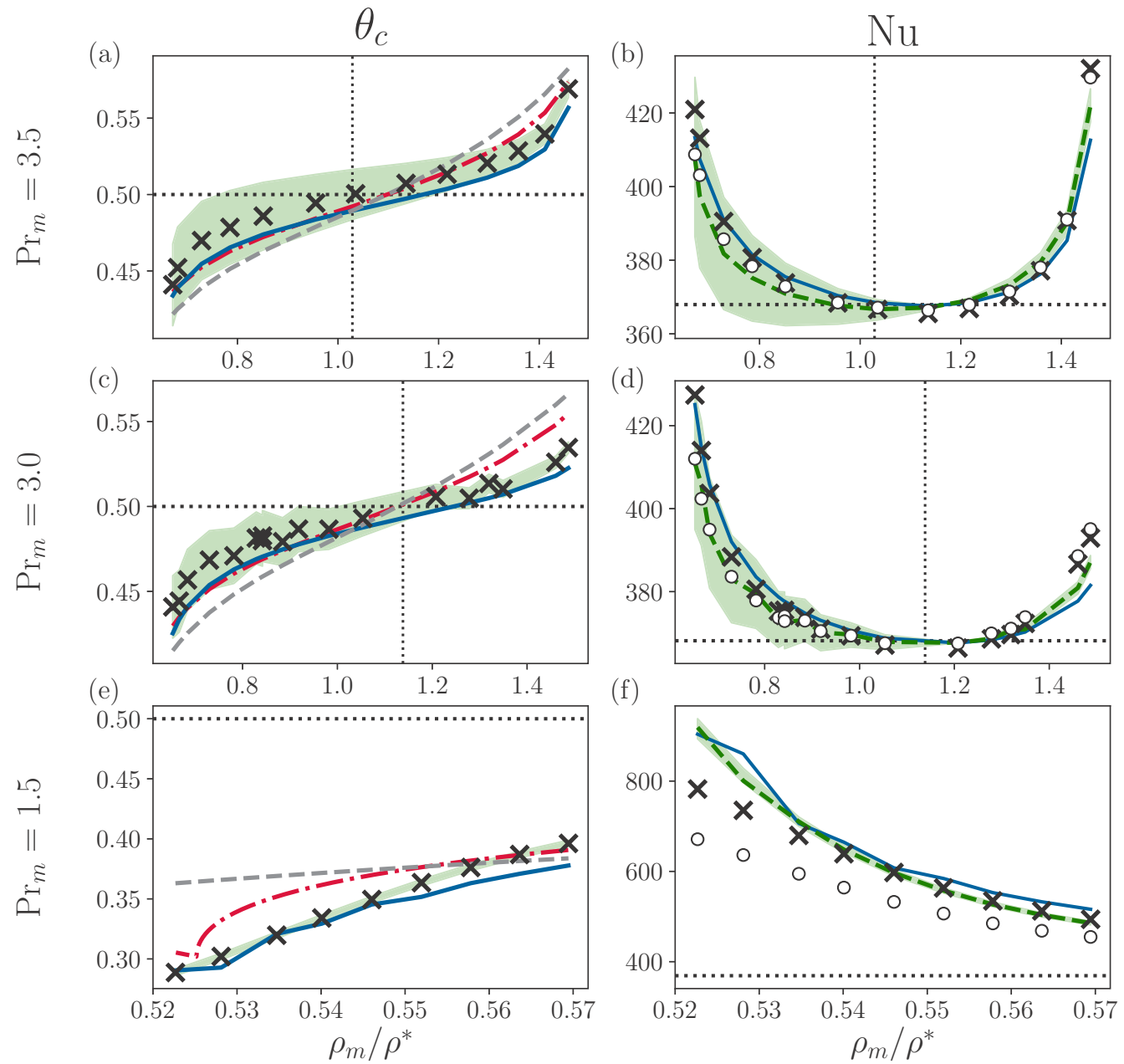

FIG. 3. Experimental results. In the left column (a), (c), (e), the measured center temperature (black crosses) is plotted with a prediction from the extended-boundary-layer theory (cyan line), the virtual-cells model (red dashed-dotted line), and the mixing zone model (gray dashed line). In the right column (b), (d), (f), measured $\mathrm{Nu}_{m}$ (black cross) and real Nusselt number (white dot) are plotted with prediction from the proposed extended GL model by using the boundary layer predicted $\theta_{c}$ (blue solid line) and using measured $\theta_{c}$ (green dashed line). The standard GL model is also plotted as the OB reference (black dotted line). The vertical line marks the measurement at which NOB effects are smallest $\left(\theta_{c}=0.5\right)$. The shaded region shows the sensitivity of $T_{c}$ measurement by displaying the effect of a $\pm 50 \mathrm{mK}$ error. Note that the Nu shown here are already corrected by $10 \%$ so that the most OB-like measurements coincide with the GL prediction.

in contrast, agrees better in all of our measurements as it takes the exact shape of $\tilde{\mathbf{X}}\left(\theta, T_{m}\right)$ into account.

In the second column of Figs. 3(b), 3(d) and 3(f), we show $\mathrm{Nu}_{m}$ (black crosses). While $\mathrm{Nu}_{m}$ is minimal close to the OB case (vertical dashed line), it is always enhanced under stronger NOB conditions, i.e., at larger (liquidlike) and smaller (gaslike) $\rho_{m} / \rho^{*}$ with heat transport enhancement of up to $100 \%$. The heat flux increase in the gaslike side was expected and was found multiple times before [14,18-21]. However, the enhancement in the liquidlike region is in contrast to measurements in liquids (water), where a small heat transport reduction $(\approx 1 \%)$ was observed [11]. We note that 
such a small reduction is within the uncertainty of our measurements. We also use circles in each plot to show the real Nusselt number, i.e., $\mathrm{Nu}=q / q_{\text {cond }}$ (as defined in [24]). We see that, while $\mathrm{Nu}$ is close to $\mathrm{Nu}_{m}$ and shows the same trend, it is visibly smaller in the gaslike regime, which is due to the positive curvature of the heat conductivity, i.e., $\partial^{2} \tilde{\Lambda} / \partial \theta^{2}>0$.

To estimate the $\mathrm{OB}$ reference for a given $\operatorname{Pr}_{m}$, we consider the experiment (or interpolations) with $\min \left(\left|\theta_{c}-0.5\right|\right)$, e.g., for $\operatorname{Pr}_{m}=3.5$ it is the one carried out at $T_{m}=58.98^{\circ} \mathrm{C}, P=49.41 \mathrm{bar}$, and $\Delta=3.10^{\circ} \mathrm{C}$. For this case, all the relevant fluid properties $\tilde{\mathbf{X}}$ are varying within $10 \%$ of their mean value and there is very little asymmetry in the experiment. There is no reason to assume that this experiment will have heat transport that is noticeably different from that under OB conditions. Nevertheless, we found that this reference heat flux is roughly $10 \%$ higher than the GL model prediction [31]. As such a deviation is easily explained by the conductive sidewalls, we will correct all measurements by this value in the following [32]. This correction is necessary, since in the next section we will compare these measurements with our own model, which includes fit parameters from the GL theory for OB convection.

\section{THEORY}

\section{A. Derivation}

In the remainder of the paper, we want to develop a quantitative model for NOB turbulent convection. For this, we first calculate the volume averaged dissipation rates from Eqs. (1) and (2),

$$
\begin{aligned}
\mathcal{E}_{u} & \equiv\left\langle\mu\left(\frac{\partial u_{i}}{\partial x_{j}}\right)^{2}\right\rangle_{V, t}=-g\left\langle\left(\rho-\rho_{c}\right) u_{z}\right\rangle_{V, t}, \\
\mathcal{E}_{T} & \equiv\left\langle\Lambda\left(\frac{\partial T}{\partial x_{j}}\right)^{2}\right\rangle_{V, t}=\frac{q \Delta}{H} .
\end{aligned}
$$

Here, we have assumed no-slip boundaries and constant pressure such that $c_{p} d T=d h$, with $h$ being the enthalpy density, expressed as a Taylor series: $h=\sum_{n=0}^{\infty} a_{n} T^{n}$. Furthermore, we only assume small pressure fluctuations such that $T \partial_{i} p \approx \partial_{i}(p T)-P_{m} \partial_{i} T$. This can be justified as the pressure variation caused by advection can be estimated as $\rho_{m} \alpha_{m} g \Delta H$, which is three orders of magnitude smaller than $P_{m}$ in our system. The exact relations for $\mathcal{E}_{u}$ and $\mathcal{E}_{T}$ are similar to the one derived for OB systems (see, e.g., [33]). Following ideas from the GL model, we separate contributions from the boundary layers and the well-mixed turbulent background,

$$
\begin{aligned}
\mathcal{E}_{u} & =\mathcal{E}_{u, \mathrm{bl}}+\mathcal{E}_{u, \mathrm{bg}}, \\
\mathcal{E}_{T} & =\mathcal{E}_{T, \mathrm{bl}}+\mathcal{E}_{T, \mathrm{bg}}
\end{aligned}
$$

This method is well-established and has been successfully applied to numerous different systems, including horizontal convection [34] and magnetoconvection [35].

First, we consider the contribution from the turbulent background (bg). We assume the background to be well mixed such that its temperature is $T=T_{c}$ with only small fluctuations. This assumption is valid when thermal plumes are excluded. This idea is inherent from the GL theory [36] that plumes are a detached boundary layer. Their dissipations are dominated by material properties, thus they scale the same as the boundary layers that will be considered in the next section. In the turbulent background region, the turbulent momentum dissipation is rather independent of the kinematic viscosity, thus

$$
\mathcal{E}_{u, \mathrm{bg}} \sim \rho_{c} \frac{U^{3}}{H}=\left(\frac{\rho_{c}}{\rho_{m}}\right) \rho_{m} \frac{v_{m}^{3}}{H^{4}} \operatorname{Re}_{m}^{3},
$$


and the turbulent thermal dissipation rate is independent of the thermal diffusivity, thus

$$
\mathcal{E}_{T, \mathrm{bg}} \sim\left(\frac{c_{p_{c}} \rho_{c}}{c_{p_{m}} \rho_{m}}\right) c_{p_{m}} \rho_{m} \kappa_{m} \frac{\Delta^{2}}{H^{2}} \operatorname{Re}_{m} \operatorname{Pr}_{m} f\left(\frac{2 a \mathrm{Nu}_{\mathrm{OB}}}{\operatorname{Re}_{\mathrm{OB}}^{1 / 2}}\right) .
$$

Here, $\mathrm{Nu}_{\mathrm{OB}}$ and $\mathrm{Re}_{\mathrm{OB}}$ are obtained by solving the standard GL model. The function $f(x)=(1+$ $\left.x^{4}\right)^{-1 / 4}$ describes the transition between regimes from small to large $\operatorname{Pr}_{m}$ [37]. Note that for now we assume this function to be identical to the one in the OB case. Here, we have furthermore neglected the other crossover function $g(x)$ from the GL model, as only turbulent systems are considered. From both expressions, one sees that the NOB effects on turbulent dissipation are only due to the shift of $T_{c}$.

In OB convection, the boundary layer and the dissipation therein are usually modeled as a Prandtl-Blasius-Pohlhausen (PBP) type [33], which assumes a self-similar velocity profile with the characteristic length $l_{u}=\sqrt{v_{m} H / U_{\infty}}$. NOB effects alter the boundary layers in two ways: (i) The characteristic velocity becomes asymmetric, as found in previous numerical $[13,16,17]$ and experimental studies [25,26], and (ii) the shape of the velocity and temperature profiles inside the boundary layers changes due to temperature-dependent fluid properties.

To address the first point, we introduce different characteristic free stream velocities $U_{\infty}$ for the top and bottom plates such that

$$
\frac{U_{\infty, \text { top }}}{b_{\text {top }}}=\frac{U_{\infty, \text { bot }}}{b_{\text {bot }}}=U=\frac{\operatorname{Re}_{m} v_{m}}{H},
$$

where $b_{\text {top }}$ and $b_{\text {bot }}$ are some undetermined factors. To describe the velocity and temperature profile in the boundary layer, we use the extended PBP, which was suggested in [14,19]. The normalized stream function $\psi$, defined such that $\sqrt{b}\left(\operatorname{Re}_{m} v_{m} / H\right) \partial_{z}\left(l_{u} \psi\right)=\tilde{\rho} u_{x}, \sqrt{b}\left(\operatorname{Re}_{m} v_{m} / H\right) \partial_{x}\left(l_{u} \psi\right)=$ $-\tilde{\rho} u_{z}$, is modeled as a function of the normalized vertical distance $\xi \equiv \sqrt{b} z / l_{u}$ as

$$
\begin{gathered}
\tilde{\mu} \frac{\partial^{3} \psi}{\partial \xi^{3}}+\left\{\frac{1}{2} \psi+\frac{\partial \tilde{\mu}}{\partial \xi}-2 \frac{\tilde{\mu}}{\tilde{\rho}} \frac{\partial \tilde{\rho}}{\partial \xi}\right\} \frac{\partial^{2} \psi}{\partial \xi^{2}}+\left\{-\frac{\psi}{2 \tilde{\rho}} \frac{\partial \tilde{\rho}}{\partial \xi}+\left[2\left(\frac{1}{\tilde{\rho}} \frac{\partial \tilde{\rho}}{\partial \xi}\right)^{2}-\frac{1}{\tilde{\rho}} \frac{\partial^{2} \tilde{\rho}}{\partial \xi^{2}}\right] \tilde{\mu}-\frac{1}{\tilde{\rho}} \frac{\partial \tilde{\rho}}{\partial \xi} \frac{\partial \tilde{\mu}}{\partial \xi}\right\} \frac{\partial \psi}{\partial \xi} \\
=0, \\
\tilde{\Lambda} \frac{\partial^{2} \theta}{\partial \xi^{2}}+\left\{\frac{1}{2} \tilde{c}_{p} \operatorname{Pr}_{m} \psi+\frac{\partial \tilde{\Lambda}}{\partial \xi}\right\} \frac{\partial \theta}{\partial \xi}=0,
\end{gathered}
$$

with boundary conditions

$$
\begin{gathered}
\psi_{\text {top } / \text { bot }}(0)=0, \\
\left(\partial_{\xi} \psi\right)_{\text {top } / \text { bot }}(0)=0, \\
\left(\partial_{\xi} \psi\right)_{\text {top } / \text { bot }}(\infty)=\tilde{\rho}_{c}, \\
\theta_{\text {top }}(0)=0, \quad \theta_{\text {bot }}(0)=1, \\
\theta_{\text {top } / \text { bot }}(\infty)=\theta_{c} .
\end{gathered}
$$

By integrating Eqs. (11) and (12) (see the Supplemental Material for details [38]), one receives expressions for the laminar viscous dissipation:

$$
\mathcal{E}_{u, \mathrm{bl}} \sim \rho_{m} \frac{v_{m}^{3}}{H^{4}} \operatorname{Re}_{m}^{5 / 2}\left(\frac{b_{\text {top }}^{5 / 2} J_{\text {top }}+b_{\text {bot }}^{5 / 2} J_{\text {bot }}}{2 J_{\mathrm{PBP}}}\right)
$$


and the thermal dissipation:

$$
\mathcal{E}_{T, \mathrm{bl}} \sim c_{p_{m}} \rho_{m} \kappa_{m} \frac{\Delta^{2}}{H^{2}} \operatorname{Re}_{m}^{1 / 2} \operatorname{Pr}_{m}^{1 / 2} f^{1 / 2}\left(\frac{2 a \mathrm{Nu}_{\mathrm{OB}}}{\operatorname{Re}_{\mathrm{OB}}^{1 / 2}}\right)\left(\frac{b_{\text {top }}^{1 / 2} K_{\text {top }}+b_{\text {bot }}^{1 / 2} K_{\mathrm{bot}}}{2 K_{\mathrm{PBP}}}\right) .
$$

Here, we have introduced the dissipation shape factors:

$$
\begin{aligned}
J & \equiv \int_{0}^{\infty} d \xi \tilde{\mu}\left[\frac{\partial}{\partial \xi}\left(\frac{\partial_{\xi} \psi}{\tilde{\rho}}\right)\right]^{2}, \\
K & \equiv \int_{0}^{\infty} d \xi \tilde{\Lambda}\left(\frac{\partial \theta}{\partial \xi}\right)^{2} .
\end{aligned}
$$

Now, we consider the correction of the buoyancy term. Under constant pressure, the average convective heat flux is $\left\langle\rho u_{z} h\right\rangle_{V}$ and the average conductive heat flux is $\int_{T_{t}}^{T_{b}} d T \Lambda / H$, so that

$$
\mathrm{Nu}_{m}=\frac{\left\langle\rho u_{z} h\right\rangle_{V}+\left(\Lambda_{m} \Delta / H\right) \int_{0}^{1} d \theta \tilde{\Lambda}}{\Lambda_{m} \Delta / H} .
$$

With (22) one can write the buoyancy term in (4) as

$$
-g\left\langle\left(\rho-\rho_{c}\right) u_{z}\right\rangle_{V}=\rho_{m} \frac{v_{m}^{3}}{H^{4}} \operatorname{Ra}_{m} \operatorname{Pr}_{m}^{-2}\left(\mathrm{Nu}_{m}-\int_{0}^{1} d \theta \tilde{\Lambda}\right)\left(\frac{-\left\langle\left(\rho-\rho_{c}\right) u_{z}\right\rangle_{V} / \alpha_{m}}{\left\langle\rho u_{z} h\right\rangle_{V} / c_{p_{m}}}\right) .
$$

Here, two new global terms appear, namely $\left\langle\left(\rho-\rho_{c}\right) u_{z}\right\rangle_{V}$ and $\left\langle\rho u_{z} h\right\rangle_{V}$. We will estimate these two terms by first-order expansion around $T_{c}$ as these two terms represent the advective mass and heat transport, respectively, which happen predominantly in the bulk. Inside the bulk, deviation of $\rho, h$ from $\rho_{c}, h_{c}$ due to temperature fluctuations can be approximated to the first order of $T^{\prime}$ as $T^{\prime}=$ $T-T_{c}$. First, $-\left\langle\left(\rho-\rho_{c}\right) u_{z}\right\rangle_{V} \approx \rho_{c} \alpha_{c}\left\langle u_{z} T^{\prime}\right\rangle_{V}$. Next, to utilize $\left\langle\rho u_{z}\right\rangle_{V}=0$, first-order expansion of $h$ around $h_{c}$ is taken first, so that $\left\langle\rho u_{z} h\right\rangle_{V} \approx h_{c}\left\langle\rho u_{z}\right\rangle_{V}+c_{p_{c}}\left\langle T^{\prime} \rho u_{z}\right\rangle_{V} \approx \rho_{c} c_{p_{c}}\left\langle u_{z} T^{\prime}\right\rangle_{V}$. This leads to

$$
-\frac{\left\langle\left(\rho-\rho_{c}\right) u_{z}\right\rangle_{V} / \alpha_{m}}{\left\langle\rho h u_{z}\right\rangle_{V} / c_{p_{m}}} \approx \frac{\left(\alpha_{c} / \alpha_{m}\right)\left\langle u_{z} T^{\prime}\right\rangle_{V}}{\left(c_{p_{c}} / c_{p_{m}}\right)\left\langle u_{z} T^{\prime}\right\rangle_{V}}=\frac{\alpha_{c} / \alpha_{m}}{c_{p_{c}} / c_{p_{m}}} .
$$

Combining Eqs. (4)-(9), (18), (19), and (23), one gets the final expression with all correction terms indicated,

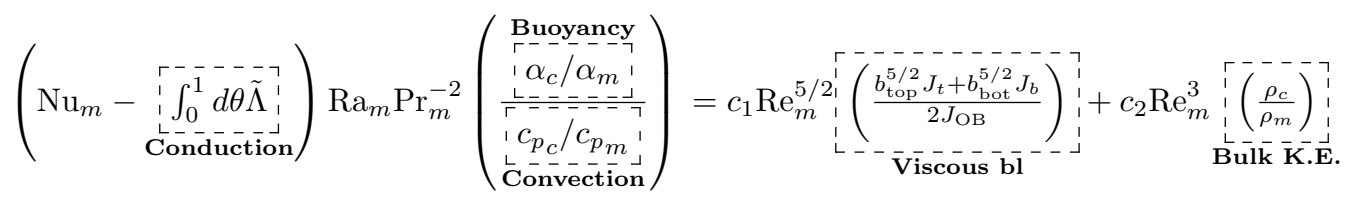

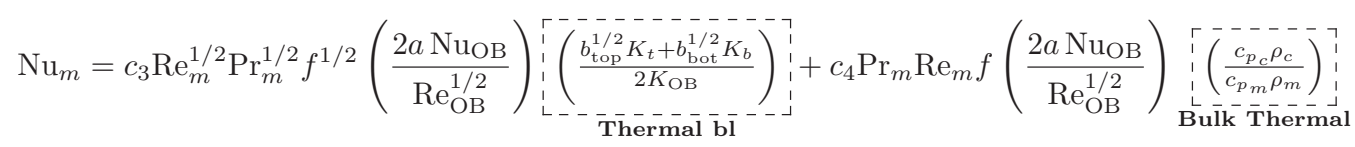

with the coefficients $c_{1}=8.05, c_{2}=1.38, c_{3}=0.487, c_{4}=0.0252$, and $a=0.922$ obtained from Stevens et al. [31]. These coefficients were obtained by fitting the GL model to available data from experiments in cylinders with aspect ratio $\Gamma=1$. While our experiments were conducted in a cell of $\Gamma=2$, we believe that the influence of the aspect ratio is small for $\Gamma \geqslant 1$, as suggested by studies 
for OB convection [39]. While we cannot exclude a stronger $\Gamma$-influence on the heat transport in NOB convection, this question is beyond the scope of this study.

To solve the set of Eqs. (25) and (26), one needs to evaluate the fluid properties at $T_{c}$ and hence one needs to know $T_{c}$. This can either be done from direct measurements or from a model such as the extended boundary-layer model ([19]). However, there are also the two unknown coefficients $b_{\text {top }}$ and $b_{\text {bot }}$, for which we currently do not have a model. However, given that the system we consider is in the GL-regime $\mathrm{IV}_{u}$, where both dissipation rates are dominated by their corresponding bulk contributions, the influence of variations of $b$ on the dissipation rates is small. To compare our model predictions with experimental data, we consider two different schemes to approximate $b_{\text {top }}$ and $b_{\text {bot: }}$ :

(i) When the boundary-layer theory accurately predicts $\theta_{c}$, we take the theoretically predicted $\theta_{c}$ (based on [14]) and have $b_{\text {top }}=b_{\text {bot }}=1$.

(ii) When the boundary-layer theory underestimates the measured $\theta_{c}$, we take the measured $\theta_{c}$ and set $b_{\text {bot }}=1$ and $b_{\text {top }}=b_{\text {bot }}\left(\left.\tilde{\Lambda} \partial_{\xi} \theta\right|_{\xi_{\text {bot }}=0}\right)^{2} /\left(\left.\tilde{\Lambda} \partial_{\xi} \theta\right|_{\xi_{\text {top }}=0}\right)^{2}$ in order to keep the heat flux conserved. For our own data, $b_{\text {top }}$ falls within the range between 0.8 and 1.2 under this scheme.

\section{B. Comparison with experimental data}

In Figs. 3(b), 3(d) and 3(f), we compare results from the model with our measurements. The blue solid line uses $T_{c}$ as predicted from the extended boundary-layer theory, and the green dashed line uses the measured $T_{c}$. As one can see, the difference between these two schemes is minimal. Furthermore, the model predictions agree well with our measured $\mathrm{Nu}_{m}$. Only for the smallest $\rho_{m} / \rho^{*}$ at $\operatorname{Pr}_{m}=1.5$ are discrepancies clearly visible. These measurements were taken very close to the critical point with very strong changes in the fluid properties due to small fluctuations in the temperature field.

To show the universality of our concept, we also test our theory against other measurements with different $\mathbf{X}(\theta), \mathrm{Ra}_{m}$, and $\operatorname{Pr}_{m}$. The studies selected in the following all show significant heat flux enhancement, while the underlying cause has not been quantitatively discussed. Ahlers et al. [19] performed experiments using pressurized ethane with $\operatorname{Pr}_{m}$ ranging from 1.79 to 3.67. The
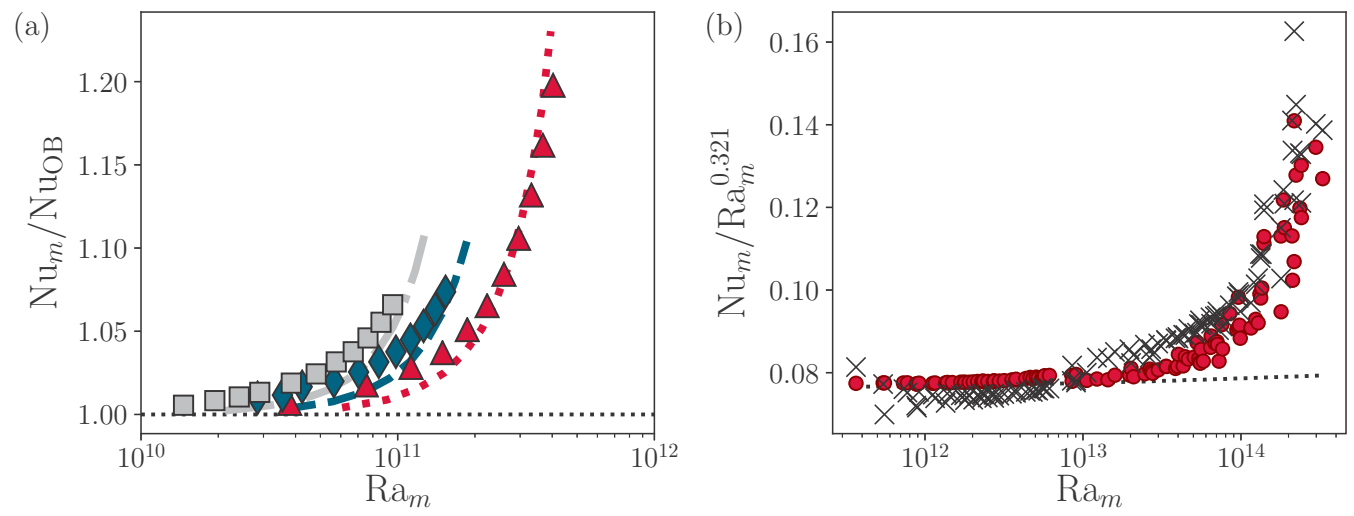

FIG. 4. (a) NOB Nusselt number enhancement in gaseous ethane at $T_{m}=40{ }^{\circ} \mathrm{C}$ plotted against the mean Rayleigh number. Measurement data for $P_{m}=1.104 P_{\text {ethane }}^{*}$ (red triangles), $P_{m}=1.062 P_{\text {ethane }}^{*}$ (teal diamonds), and $P_{m}=0.991 P_{\text {ethane }}^{*}$ (gray squares) are adapted from [19]. Model predictions by the proposed model for $P_{m}=$ $1.104 P_{\text {ethane }}^{*}$ (red dotted line), $P_{m}=1.062 P_{\text {ethane }}^{*}$ (teal dashed line), and $P_{m}=0.991 P_{\text {ethane }}^{*}$ (gray dashed-dotted line) are plotted for comparison. (b) Compensated mean Nusselt number in cryogenic helium vs mean Rayleigh number measured by [23] (black cross) and our model prediction (red dots). Dotted line: GL prediction for OB reference. The $\mathrm{Ra}_{m}$ and $\mathrm{Nu}_{m}$ have been recalculated using REFPROP 9.1 [29]. 
convection experiments were carried out at $T_{m}=40^{\circ} \mathrm{C}$ in a $\Gamma=1$ cell. Three sets of experiments were performed at different pressure, and the degree of NOB effects was controlled by changing the applied temperature differences $\Delta$. In Fig. 4(a), we show that our model agrees well with their experimental data. We used scheme (1) to obtain $T_{c}$ as it is claimed that the boundary-layer theory predicts $T_{c}$ reasonably well in the paper.

Urban et al. [23] performed experiments using pressurized cryogenic helium with $\operatorname{Pr}_{m}$ ranging from 0.68 to 11.29 in an $\Gamma=1$ cell. $T_{m}, P_{m}$, and $\Delta$ are varied to achieve measurements in over two decades of $\mathrm{Ra}_{m}$. Detailed experimental conditions can be found in the Appendix of the paper. In Fig. 4(b), we show that our model also agrees well in such a wide range of $\operatorname{Pr}_{m}$ and $\operatorname{Ra}_{m}$. We used scheme (2) to obtain $T_{c}$ as we found that the boundary-layer theory did not provide accurate $T_{c}$ shifting.

Apart from these two studies, we also tested our model with studies on ordinary fluid such as water [11]. While our current model predicts that NOB effects do not lead to large heat flux changes, we do not believe that the effect is well captured. This is because despite the drastic $T_{c}$ change, its effect on $c_{p}$ and $\rho$ is negligible. In such a case, secondary effects, namely the Pr number transition [described by $f(x)$ in the GL model], and the relative characteristic velocity at the top and bottom plate (described by $b_{t}$ and $b_{b}$ ) become important.

\section{CONCLUSION}

In this paper, we propose an experimental scheme to study the influence of NOB effects on the heat flux in turbulent Rayleigh-Bénard convection at a constant Rayleigh (Ra) and Prandtl number (Pr). This is done by using compressed $\mathrm{SF}_{6}$ above its critical point, where it is neither a liquid nor a gas. There, one can find isolines in the $T-P$ phase space, along which the Prandtl number is constant. The mean Rayleigh number can be held constant as well by choosing the temperature difference between bottom and top $\Delta$ appropriately. Thus one can vary the differences in the fluid properties between bottom and top while keeping $\operatorname{Ra}_{m}$ and $\operatorname{Pr}_{m}$ constant. In particular, one can study liquid- and gaslike fluids at large and small $\rho$, which show qualitatively different NOB effects.

In doing so, we have explored thoroughly how the temperature-dependent material properties, i.e., $\tilde{\mathbf{X}}(\theta)$, affect the midheight temperature $T_{c}$ and the global heat flux $\mathrm{Nu}_{m}$ without the influence of the change of $\mathrm{Ra}_{m}$ and $\operatorname{Pr}_{m}$. We found that $T_{c}$ is smaller than the average temperature $T_{m}$ for gaslike fluids and larger for liquidlike fluids. This finding is in good agreement with previous measurements in liquids and gases. However, we also found that the vertical heat flux does not show a monotonic behavior. Instead, we have observed a strong heat transport enhancement for both the liquid- and the gaslike region, by up to $112 \%$.

Furthermore, we have addressed the long-standing question of what causes this significant heat flux enhancement by developing a predictive extended Grossmann-Lohse model for the heat transport under NOB conditions. Our model predictions not only agree well with our own experimental measurements, but also with previous measurements in pressurised ethane and cryogenic helium for a wide range of $\operatorname{Ra}_{m}$ and $\operatorname{Pr}_{m}$. Our model suggests that the heat capacity $c_{p}$ and density $\rho$ in the midheight, which is often overlooked in existing boundary-layer-based theories, significantly affect the global heat flux. We believe that our model is useful for applications and experiments performed under a high variation of $\rho$ and $c_{p}$.

\section{ACKNOWLEDGMENTS}

We thank Lucia Wesenberg for building the high-precision pressure regulation system. V.V. acknowledges financial support from the European High-Performance Infrastructure in Turbulence (EuHIT). 
[1] A. Oberbeck, Über die wärmeleitung der flüssigkeiten bei berücksichtigung der strömungen infolge von temperaturdifferenzen, Ann. Phys. Chem. 243, 271 (1879).

[2] J. Boussinesq, Theorie Analytique de la Chaleur (Gauthier-Villars, Paris, 1903), Vol. 2.

[3] E. A. Spiegel and G. Veronis, On the Boussinesq approximation for a compressible fluid, Astrophys. J. 131, 442 (1960).

[4] G. Ahlers, S. Grossmann, and D. Lohse, Heat transfer and large scale dynamics in turbulent RayleighBénard convection, Rev. Mod. Phys. 81, 503 (2009).

[5] S. Hanasoge, L. Gizon, and K. R. Sreenivasan, Seismic sounding of convection in the sun, Annu. Rev. Fluid Mech. 48, 191 (2016).

[6] V. Valori, Rayleigh-Bénard convection of a supercritical fluid: PIV and heat transfer study, Ph.D. thesis, TU Delft (2018).

[7] I. L. Pioro, R. B. Duffey, P. L. Kirillov, and R. Panchal, 1 - Introduction: A survey of the status of electricity generation in the world, Handbook of Generation IV Nuclear Reactors, edited by Igor L. Pioro, Woodhead Publishing Series in Energy (Woodhead Publishing, 2016), pp. 1-34.

[8] Understanding and Prediction of Thermohydraulic Phenomena Relevant to Supercritical Water Cooled Reactors (SCWRs), TECDOC Series No. 1900 (International Atomic Energy Agency, Vienna, 2020).

[9] F. Busse, The stability of finite amplitude cellular convection and its relation to an extremum principle, J. Fluid Mech. 30, 625 (1967).

[10] D. D. Gray and A. Giorgini, The validity of the Boussinesq approximation for liquids and gases, Int. J. Heat Mass Trans. 19, 545 (1976).

[11] G. Ahlers, E. Brown, F. F. Araujo, D. Funfschilling, S. Grossmann, and D. Lohse, Non-OberbeckBoussinesq effects in strongly turbulent Rayleigh-Bénard convection, J. Fluid Mech. 569, 409 (2006).

[12] J. Zhang, S. Childress, and A. Libchaber, Non-Boussinesq effect: Thermal convection with broken symmetry, Phys. Fluids 9, 1034 (1997).

[13] K. Sugiyama, E. Calzavarini, S. Grossmann, and D. Lohse, Flow organization in two-dimensional nonOberbeck-Boussinesq Rayleigh-Bénard convection in water, J. Fluid Mech. 637, 105 (2009).

[14] G. Ahlers, E. Calzavarini, F. F. Araujo, D. Funfschilling, S. Grossmann, D. Lohse, and K. Sugiyama, Non-Oberbeck-Boussinesq effects in turbulent thermal convection in ethane close to the critical point, Phys. Rev. E 77, 046302 (2008).

[15] A. D. Demou and D. G. Grigoriadis, Direct numerical simulations of Rayleigh-Bénard convection in water with non-Oberbeck-Boussinesq effects, J. Fluid Mech. 881, 1073 (2019).

[16] S. Horn, O. Shishkina, and C. Wagner, On non-Oberbeck-Boussinesq effects in three-dimensional Rayleigh-Bénard convection in glycerol, J. Fluid Mech. 724, 175 (2013).

[17] S. Horn and O. Shishkina, Rotating non-Oberbeck-Boussinesq Rayleigh-Bénard convection in water, Phys. Fluids 26, 055111 (2014).

[18] X.-Z. Wu and A. Libchaber, Non-Boussinesq effects in free thermal convection, Phys. Rev. A 43, 2833 (1991).

[19] G. Ahlers, F. Fontenele Araujo, D. Funfschilling, S. Grossmann, and D. Lohse, Non-OberbeckBoussinesq Effects in Gaseous Rayleigh-Bénard Convection, Phys. Rev. Lett. 98, 054501 (2007).

[20] S. Weiss, X. He, G. Ahlers, E. Bodenschatz, and O. Shishkina, Bulk temperature and heat transport in turbulent Rayleigh-Bénard convection of fluids with temperature-dependent properties, J. Fluid Mech. 851, 374 (2018).

[21] Z.-H. Wan, Q. Wang, B. Wang, S.-N. Xia, Q. Zhou, and D.-J. Sun, On non-Oberbeck-Boussinesq effects in Rayleigh-Bénard convection of air for large temperature differences, J. Fluid Mech. 889, A10 (2020).

[22] P. Urban, P. Hanzelka, T. Kralik, V. Musilova, A. Srnka, and L. Skrbek, Effect of Boundary Layers Asymmetry on Heat Transfer Efficiency in Turbulent Rayleigh-Bénard Convection at Very High Rayleigh Numbers, Phys. Rev. Lett. 109, 154301 (2012).

[23] P. Urban, P. Hanzelka, V. Musilová, T. Králík, M. La Mantia, A. Srnka, and L. Skrbek, Heat transfer in cryogenic helium gas by turbulent Rayleigh-Bénard convection in a cylindrical cell of aspect ratio 1 , New J. Phys. 16, 053042 (2014). 
[24] O. Shishkina, S. Weiss, and E. Bodenschatz, Conductive heat flux in measurements of the Nusselt number in turbulent Rayleigh-Bénard convection, Phys. Rev. Fluids 1, 062301 (2016).

[25] V. Valori, G. Elsinga, M. Rohde, M. Tummers, J. Westerweel, and T. van der Hagen, Experimental velocity study of non-Boussinesq Rayleigh-Bénard convection, Phys. Rev. E 95, 053113 (2017).

[26] V. Valori, G. E. Elsinga, M. Rohde, J. Westerweel, and T. H. J. J. van der Hagen, Particle image velocimetry measurements of a thermally convective supercritical fluid, Exp. Fluids 60, 143 (2019).

[27] G. Ahlers, Effect of sidewall conductance on heat-transport measurements for turbulent Rayleigh-Bénard convection, Phys. Rev. E 63, 015303 (2000).

[28] P. Roche, B. Castaing, B. Chabaud, B. Hebral, and J. Sommeria, Side wall effects in Rayleigh-Bénard experiments, Eur. Phys. J. 24, 405 (2001).

[29] E. Lemmon, M. Huber, and M. McLinden, NIST standard reference database 23, reference fluid thermodynamic and transport properties (refprop), version 9.0, National Institute of Standards and Technology, R1234yf. fld file dated December 22 (2010).

[30] This is due in particular to the highly irregular shape of $\tilde{\mathbf{X}}(\theta)$.

[31] R. J. Stevens, E. P. van der Poel, S. Grossmann, and D. Lohse, The unifying theory of scaling in thermal convection: the updated prefactors, J. Fluid Mech. 730, 295 (2013).

[32] Measurements in ethane with a similar $(\Gamma=1)$-cell [14] show a $25 \%$ larger Nu under OB-condition than the GL-model. Taking into account that our cell is of $\Gamma=2$, the influence of the sidewall is smaller and thus $\mathrm{Nu}$ is only $10 \%$ larger than the GL model.

[33] S. Grossmann and D. Lohse, Scaling in thermal convection: a unifying theory, J. Fluid Mech. 407, 27 (2000).

[34] O. Shishkina, S. Grossmann, and D. Lohse, Heat and momentum transport scalings in horizontal convection, Geophys. Res. Lett. 43, 1219 (2016).

[35] T. Zurner, W. Liu, D. Krasnov, and J. Schumacher, Heat and momentum transfer for magnetoconvection in a vertical external magnetic field, Phys. Rev. E 94, 043108 (2016).

[36] S. Grossmann and D. Lohse, Fluctuations in turbulent Rayleigh-Bénard convection: the role of plumes, Phys. Fluids 16, 4462 (2004).

[37] S. Grossmann and D. Lohse, Thermal Convection for Large Prandtl Numbers, Phys. Rev. Lett. 86, 3316 (2001).

[38] See Supplemental Material at http://link.aps.org/supplemental/10.1103/PhysRevFluids.5.103502 for a derivative of the dissipation rates inside the boundary layer, as well as a table with the experimental condition for which experiments were performed.

[39] R. J. Stevens, A. Blass, X. Zhu, R. Verzicco, and D. Lohse, Turbulent thermal superstructures in RayleighBénard convection, Phys. Rev. Fluids 3, 041501 (2018). 